\title{
Photothermal ablation of inflammatory breast cancer tumor emboli using plasmonic gold nanostars
}

\author{
Bridget M Crawford $1,2, *$ \\ Ronnie L Shammas ${ }^{3, *}$ \\ Andrew M Fales ${ }^{1,2}$ \\ David A Brown ${ }^{4}$ \\ Scott T Hollenbeck ${ }^{4}$ \\ Tuan Vo-Dinh ${ }^{1,2,5}$ \\ Gayathri R Devi ${ }^{6,7}$
}

'Fitzpatrick Institute for Photonics, Duke University, ${ }^{2}$ Department of Biomedical Engineering, Duke University, ${ }^{3}$ Duke University School of Medicine, ${ }^{4}$ Department of Surgery, Division of Plastic, Maxillofacial, and Oral Surgery, Duke University Medical Center, ${ }^{5}$ Department of Chemistry, Duke University, ${ }^{6}$ Department of Surgery, Division of Surgical Sciences, ${ }^{7}$ Duke Cancer Institute, Women's Cancer Program, Duke University School of Medicine, Durham, NC, USA

*These authors contributed equally to this work
This article was published in the following Dove Press journal:

International Journal of Nanomedicine

26 August 2017

Number of times this article has been viewed

\begin{abstract}
Inflammatory breast cancer (IBC) is rare, but it is the most aggressive subtype of breast cancer. IBC has a unique presentation of diffuse tumor cell clusters called tumor emboli in the dermis of the chest wall that block lymph vessels causing a painful, erythematous, and edematous breast. Lack of effective therapeutic treatments has caused mortality rates of this cancer to reach $20 \%-30 \%$ in case of women with stage III-IV disease. Plasmonic nanoparticles, via photothermal ablation, are emerging as lead candidates in next-generation cancer treatment for site-specific cell death. Plasmonic gold nanostars (GNS) have an extremely large two-photon luminescence cross-section that allows real-time imaging through multiphoton microscopy, as well as superior photothermal conversion efficiency with highly concentrated heating due to its tip-enhanced plasmonic effect. To effectively study the use of GNS as a clinically plausible treatment of IBC, accurate three-dimensional (3D) preclinical models are needed. Here, we demonstrate a unique in vitro preclinical model that mimics the tumor emboli structures assumed by IBC in vivo using IBC cell lines SUM149 and SUM190. Furthermore, we demonstrate that GNS are endocytosed into multiple cancer cell lines irrespective of receptor status or drug resistance and that these nanoparticles penetrate the tumor embolic core in 3D culture, allowing effective photothermal ablation of the IBC tumor emboli. These results not only provide an avenue for optimizing the diagnostic and therapeutic application of GNS in the treatment of IBC but also support the continuous development of $3 \mathrm{D}$ in vitro models for investigating the efficacy of photothermal therapy as well as to further evaluate photothermal therapy in an IBC in vivo model.
\end{abstract}

Keywords: inflammatory breast cancer, photothermal therapy, hyperthermia, plasmonics, gold nanostars, nanoparticles

\section{Introduction}

IBC is the most lethal and aggressive breast cancer subtype with a unique clinicopathological presentation. ${ }^{1-3}$ Patients diagnosed with IBC often present at an advanced stage with lymph node involvement, of whom $30 \%$ already have distant metastases..$^{4-6}$ The high morbidity rate and aggressive nature of IBC compared to other breast cancer subtypes is thought to be attributed to the presence of tumor emboli in the dermal lymphatics. Invasion of lymphatic vessels by these tumor cell clusters results in a rapid metastatic progression of this disease. ${ }^{6-8}$ Developing an effective treatment for IBC is challenging due to the difficulties associated with studying the lymphovascular tumor emboli in vitro. Standard 2D cell culture techniques do not accurately recapitulate the tumor microenvironment of IBC, further complicating the successful translation of cancer therapeutics from an in vitro to an in vivo model. ${ }^{8,9}$
Correspondence: Gayathri R Devi

Department of Surgery, Duke University School of Medicine, BOX 2606, Durham, NC 2770I, USA

Tel+19196680410

Email gayathri.devi@duke.edu

Tuan Vo-Dinh

Duke University, 1427 CIEMAS, BOX 9028I, Durham, NC 27708, USA

Tel + I 9196608520

Email tuan.vodinh@duke.edu 
Tumor-targeting nanomaterials have great potential in significantly improving the efficacy of anti-cancer therapeutics while limiting systemic toxicity. ${ }^{10,11}$ Due to the inert nature of gold, gold nanoparticles are one of the most promising nanoparticle variants for cancer treatment. Several gold nanoparticles that exhibit low cytotoxicity have been developed (including nanorods, nanoshells, nanocages, and nanostars) with properties that vary with particle size and shape. ${ }^{12}$ Noble metal nanoparticles are quite useful for cellular tracking owing to their two-TPL cross-sections. ${ }^{13} \mathrm{TPL}$ involves the absorption of photons and emission from the recombination of excited electrons in the $s p$ conduction band with $d$ band holes. ${ }^{14}$ Nanorods and nanobranches demonstrate a significantly greater degree of luminescence compared to nanospheres, thereby improving cellular tracking. ${ }^{12}$ GNS fall within the family of nanobranches, as they are branched nanoparticles with multiple protruding spikes that exhibit a greater localized electric field associated with their surface plasmon resonance. ${ }^{15}$ Our lab has developed a surfactant-free method of synthesizing GNS that does not require cytotoxic chemicals and allows for in vitro intracellular accumulation of GNS via macropinocytosis following conjugation with the transactivator of transcription (TAT) peptide. ${ }^{16}$ The higher photon-to-heat conversion efficiency of GNS, which can be tuned to have spectral absorption in the near-infrared region where tissue absorbs least, makes GNS superior in biological imaging and nanotherapeutic capabilities as compared to other commonly used nanoparticles. ${ }^{14,15,17-19}$

With regard to in vivo translation, GNS can easily penetrate tumor vasculature via EPR effect that originates from the inherently leaky tumor vasculature. ${ }^{20,21}$ Furthermore, once delivered to the tumor microenvironment, there is minimal clearance of nanoparticles that range from 10 to $100 \mathrm{~nm}$ in size due to lack of an efficient lymphatic system..$^{21,22}$ Once retained in tumor tissues, these nanoparticles can generate heat upon stimulation by an NIR laser due to their localized surface plasmon effect. ${ }^{16}$ This heating allows for hyperthermic therapy and cellular ablation simply by the application of a cutaneous laser.

With regard to therapeutic use of nanoparticles, the translation of test results from 2D monolayer cell culture to in vivo conditions remains questionable. ${ }^{23-26}$ There are many inherent differences in the sensitivity and efficacy of chemotherapeutics between $2 \mathrm{D}$ and $3 \mathrm{D}$ culture models. ${ }^{27-29}$ Using a 2D model, it is difficult to elucidate the subtleties of the nanoparticles' interaction with tumor biology; for example, the depth of penetrance into the tumor structure, differences in localization and cellular uptake, and efficacy of photothermal treatment cannot be fully appreciated when using a monolayer system. Furthermore, 3D culture models provide a platform that more closely mimics the complex and dynamic tumor microenvironment compared to conventional 2D cultures. ${ }^{9,30,31}$ In addition, this model allows to manipulate and study tumor emboli following exposure to nanoparticles in a way that is not possible in vivo, owing to the difficulty in isolating lymphovascular emboli from in vivo models of IBC. The ability to combine the study of therapeutic nanoparticles with the use of 3D in vitro tumor models offers an ideal platform to aid in the development of nanotherapeutics to target drug-resistant cancers, like IBC, and also allows to optimize nanotherapeutic techniques prior to conducting studies in an in vivo model.

To this end, we have developed a noninvasive photothermal application implementing GNS as a novel therapeutic agent for the treatment of IBC using a 3D in vitro model. GNS are among the most promising nanoparticles for cancer therapy due to their ease of delivery, sensitive tracking capability, superior photothermal effects, and potential for multimodal imaging. ${ }^{20,32,33}$ In this study, GNS were used in combination with a unique in vitro model of IBC to assess the therapeutic effect of GNS-mediated hyperthermal therapy in 3D culture. This is the first study to analyze the effects of nanotherapeutics using an in vitro model that mimics tumor biology of IBC.

\section{Materials and methods \\ Cell lines and culture conditions}

SUM149 (inflammatory ductal carcinoma) and SUM190 (primary human inflammatory luminal breast carcinoma) cell lines were obtained from Asterand, Inc., and cultured as previously described. ${ }^{34}$ rSUM149 cells, which exhibit multidrug resistance, ${ }^{35}$ were synthesized in our laboratory from SUM149 cells and cultured in a manner similar to SUM149 cells with $7.5 \mu$ M GW583340 (Sigma-Aldrich, St Louis, MO, USA) added a day after splitting for each passage as described previously. ${ }^{35,36}$ BT474M1 (invasive ductal carcinoma) provided by Dr Timothy Clay at Duke University, was cultured in Dulbecco's Modified Eagle's Medium/F12 (Sigma-Aldrich) supplemented with 10\% fetal bovine serum (Sigma-Aldrich) and 1\% penicillin/streptomycin. MDA-MB231 (breast adenocarcinoma), purchased from ATCC, was grown in RPMI medium (Gibco) supplemented with $10 \%$ fetal bovine serum (Sigma-Aldrich) and $1 \%$ penicillin/streptomycin. All cell lines were maintained at $37^{\circ} \mathrm{C}$ in a humidified 
atmosphere of 5\% $\mathrm{CO}_{2}$, subcultured in T-25 and T-75 flasks and supplemented with fresh media every 2-3 days. All samples were grown to $\sim 80 \%$ confluency before use. Cells were detached using $0.25 \%$ trypsin-EDTA (Gibco).

\section{GNS preparation}

All chemicals were purchased from Sigma-Aldrich and used as received unless noted otherwise. GNS were prepared by a surfactant-free method. ${ }^{16}$ Citrate-capped gold seeds were prepared by adding $15 \mathrm{~mL}$ of $1 \%$ trisodium citrate to $100 \mathrm{~mL}$ of boiling $\mathrm{HAuCl}_{4}$ solution $(1 \mathrm{mM})$ under vigorous stirring for 15 minutes. The solution was cooled to room temperature, filtered with a $0.22 \mu \mathrm{m}$ nitrocellulose membrane, and stored at $4{ }^{\circ} \mathrm{C}$. GNS were prepared by simultaneously mixing $1 \mathrm{~mL}$ of $3 \mathrm{mM} \mathrm{AgNO}_{3}$ and $500 \mu \mathrm{L}$ of $0.1 \mathrm{M}$ ascorbic acid to $100 \mathrm{~mL}$ of $0.25 \mathrm{mM} \mathrm{HAuCl}_{4}$ containing $\mathrm{HCl}$ $(100 \mu \mathrm{L}, 1 \mathrm{~N})$ and citrate gold seeds $\left(1 \mathrm{~mL}, \mathrm{OD}_{520}: 2.8\right)$ under vigorous stirring. For particle stabilization, $5 \mu \mathrm{M}$ of SHPEG $_{5000}\left(O-\right.$-2-(3mercaptopropionylamino) ethyl]- $O^{\prime}$ methylpolyethlene-glycol, molecular weight 5,000) was added to freshly synthesized GNS and allowed to mix for 30 minutes, followed by two centrifugal washes (3,500 rcf). Lastly, TATGNS were prepared by mixing a final solution of $100 \mu \mathrm{M}$ of cysteine-TAT-peptide (residues 49-57, sequence Arg-LysLys-Arg-Arg-Arg-Gln-Arg-Cys-CONH ${ }_{2}$; SynBioSci, Livermore, CA, USA) with $1 \mathrm{nM}$ of PEG-GNS solution for 48 hours at $4^{\circ} \mathrm{C}$ followed by two centrifugal washes in ethanol.

\section{GNS labeling of breast cancer cell lines}

GNS solutions were prepared for cellular incubation by resuspending in PBS at $1.0 \mathrm{nM}$. GNS were added to the appropriate tissue culture flask or plate containing media at final concentration of $0.15 \mathrm{nM}$. Cells were incubated with the GNS-containing solution for 24 hours in a tissue culture flask. After incubation, cells were washed twice with PBS to remove any residual nanoparticle solution prior to experimental plating.

\section{MPM imaging}

All MPM images were taken using a multiphoton microscope (Olympus FV1000; Olympus America, Center Valley, PA, USA) at the Light Microscopy Core Facility, Duke University. Microscopic imaging was carried out using a femtosecond Ti:Sapphire laser (Chameleon Vision II; Coherent, Santa Clara, CA, USA) with tunable wavelength ranging from 680 to $1,080 \mathrm{~nm}, 140$ femtosecond (fsec) pulse width, and $80 \mathrm{MHz}$ repetition rate. The laser beam was focused through a $25 \times 1.05$ NA water-immersion objective (XLPL25XWMP; Olympus America). Images were taken under $800 \mathrm{nM}$ excitation and $3.7 \mathrm{~mW}$ unless otherwise noted. All images were collected and reconstructed using ImageJ. ${ }^{37}$

\section{MTT cellular proliferation and resazurin cell viability assays}

Cellular proliferation was determined by MTT (SigmaAldrich) assay and cellular proliferation at varying GNS concentrations and incubation times was compared. In this assay, MTT is reduced by metabolically active cells, which results in purple formazan that can be quantified by spectrophotometry. GNS at concentrations of $0.05,0.1,0.15$, and $0.2 \mathrm{nM}$ were incubated with SUM149 cells at various time periods of 1, 6, 12, and 24 hours. SUM149 cells were plated in a 96-well plate and incubated overnight. The respective concentrations of GNS were then added to the wells as per the aforementioned incubation time. After each time point, the GNS-containing media was aspirated from the wells, washed twice with PBS, and supplemented with fresh media. After all time points were complete, $20 \mu \mathrm{L}$ of MTT solution was added to each well for 2 hours at $37^{\circ} \mathrm{C}$. After incubation, the MTT solution was discarded and $100 \mu \mathrm{L}$ of DMSO was added to dissolve the formazan crystals. The absorbance was then measured using a spectrophotometer (FLUOstar Optima, BMG Biotech) with a $575 \mathrm{~nm}$ filter.

For cytotoxicity assay, SUM149 cells were incubated with $0.05,0.1,0.15$, and $0.2 \mathrm{nM}$ of GNS at various time periods of 1, 6, 12, and 24 hours. SUM149 cells were plated in a 96-well plate and incubated overnight. The respective concentrations of GNS were then added to the wells as per the aforementioned incubation time. After each time point, the GNS-containing media was aspirated from the wells, washed twice with PBS, and supplemented with fresh media. After all time points were complete, $20 \mu \mathrm{L}$ of the CellTiter-Blue ${ }^{\mathbb{R}}$ (resazurin assay; Promega, Madison, WI, USA) reagent was added to each well and the plates were incubated for 2 hours. Resazurin (blue, nonfluorescent) is reduced by live cells to resorufin (pink, fluorescent). The fluorescence intensity of resorufin was then measured using a plate reader (FLUOstar Omega; BMG LABTECH) to determine the percent of cell viability relative to the unlabeled controls.

\section{TEM imaging of cells}

For TEM, fixed cells were stained with $\mathrm{OsO}_{4}$ and uranyl acetate followed by ethanol series dehydration and resin fixation. Ultrathin sections were cut by an ultramicrotome, 
mounted on copper grids, stained with uranyl acetate/lead citrate and imaged using a Fei Tecnai $\mathrm{G}^{2}$ Twin at $80 \mathrm{kV}$.

\section{Quantification of nanoparticles per cell using ICP-OES}

ICP-OES was used to quantitatively analyze nanoparticle uptake by SUM149 cell lines. Nanoparticle-containing cells were digested in a solution of Aqua Regia and diluted with deionized water before being analyzed by ICP-OES at the Environmental and Agricultural Testing Service Laboratory, North Carolina State University. Experimental samples were compared to a control sample of known GNS content.

\section{In vitro photothermal therapy of cancer cell lines in monoculture}

Photothermal therapy was applied to GNS-labeled cancer cell lines following particle incubation as described earlier. GNSlabeled cells were seeded in $35 \mathrm{~mm}$ Petri dishes, incubated overnight, washed with PBS twice to remove unattached cells, and supplemented with fresh media. For photothermal therapy, cells on a heated multiphoton microscope stage $\left(37^{\circ} \mathrm{C}\right)$ were exposed to Ti:Sapphire laser at a wavelength of $800 \mathrm{~nm}$ for 3 minutes, with output powers of 2.19, 3.7, or 9.14 $\mathrm{mW}$. Cells cultured without GNS were used as controls but received the same laser irradiation. Immediately after treatment, cells were examined under a fluorescence microscope using fluorescein diacetate and propidium iodide, which stain live cells green and dead cells red, respectively.

\section{Tumor emboli preparation}

IBC cell lines SUM149, rSUM149, and SUM190 were used in tumor emboli models as performed in previous studies. ${ }^{7}$ Briefly, cells were seeded onto ultra-low attachment plates (Corning) at appropriate seeding densities and suspended in culture medium supplemented with $2.25 \%$ PEG-8000 (Sigma-Aldrich). After suspension, cells were placed on a belly dance shaker (IBI Scientific $($ ) and allowed to rock at $40 \mathrm{rpm}$. The cells were incubated in these suspension conditions for up to 96 hours prior to experimental use.

\section{GNS labeling of tumor emboli}

For tumor emboli models, GNS was added to the IBC tumor emboli culture after 96 hours of embolic maturation. Tumor emboli were then allowed to incubate with GNS for 24 hours prior to experimental use.

\section{H\&E staining of tumor emboli}

For all histological staining of tumor emboli, a gelatin embedding method was used to prepare the samples. For gelatin embedding, samples were placed in $5 \mathrm{~mL}$ of $5 \%(\mathrm{w} / \mathrm{w})$ porcine-derived gelatin (Sigma-Aldrich) solution with $5 \%(\mathrm{w} / \mathrm{w})$ sucrose that was prewarmed to $45^{\circ} \mathrm{C}$ for 2 hours, followed by immersion in an acetone and dry-ice bath until frozen as previously described. ${ }^{38}$ Samples were then cryosectioned at a thickness of $20 \mu \mathrm{m}$. Prior to histological staining, all samples were stored at $-80^{\circ} \mathrm{C}$. Standard $\mathrm{H} \& \mathrm{E}$ staining was used to confirm the acquisition of tumor emboli following sectioning.

\section{In vitro photothermal therapy of GNS-labeled tumor emboli}

The effects of photothermal therapy on GNS-labeled SUM149, rSUM149, and SUM190 tumor emboli were assessed. Tumor emboli were cultured in 96-well plates for 96 hours and then incubated with $0.15 \mathrm{nM}$ GNS for 24 hours prior to photothermal therapy. For photothermal response validation, a continuous $808 \mathrm{~nm}$ diode laser was focused on the tumor emboli. Emboli were kept on a $37^{\circ} \mathrm{C}$ heating stage and exposed to the laser for 3 minutes at power densities of $9.375,5$, and $2.18 \mathrm{~W} / \mathrm{cm}^{2}$ (the spot size of laser beam being $33 \mathrm{~mm}^{2}$ ). Samples cultured without GNS were used as controls but received the same laser irradiation. After 1 day, tumor emboli were examined by a dead-cell staining procedure using propidium iodide.

\section{Results}

\section{GNS display robust and sustained} cytoplasmic endocytosis within IBC cells

The GNS synthesized were proved to be stable in cell culture medium (Figure S1). TEM was used to show the morphology of a single GNS (Figure 1A), as well as the abundance and accumulation patterns of GNS within IBC cells following 24-hour incubation, using SUM149 as a model cell line (Figure 1B-D). These images revealed abundant cytoplasmic accumulation of GNS, with most of the intracellular nanoparticle accumulation confined to the vesicles of the cells (Figure 1A-C). In addition, these images confirmed that the accumulation of GNS was restricted to the extranuclear space of the cells. ICP-OES was pursued to further analyze the uptake patterns of GNS in IBC cells. Quantitative analysis revealed that the number of GNS in the SUM149 cell line gradually increased with incubation times of $1,4,8$, and 24 hours (Figure 1D). Moreover, the number of nanoparticles per cell did not reach a plateau within the 24-hour incubation period, suggesting that the uptake efficiency and retention of GNS did not exhibit an intracellular decay in terms of nanoparticle load. 

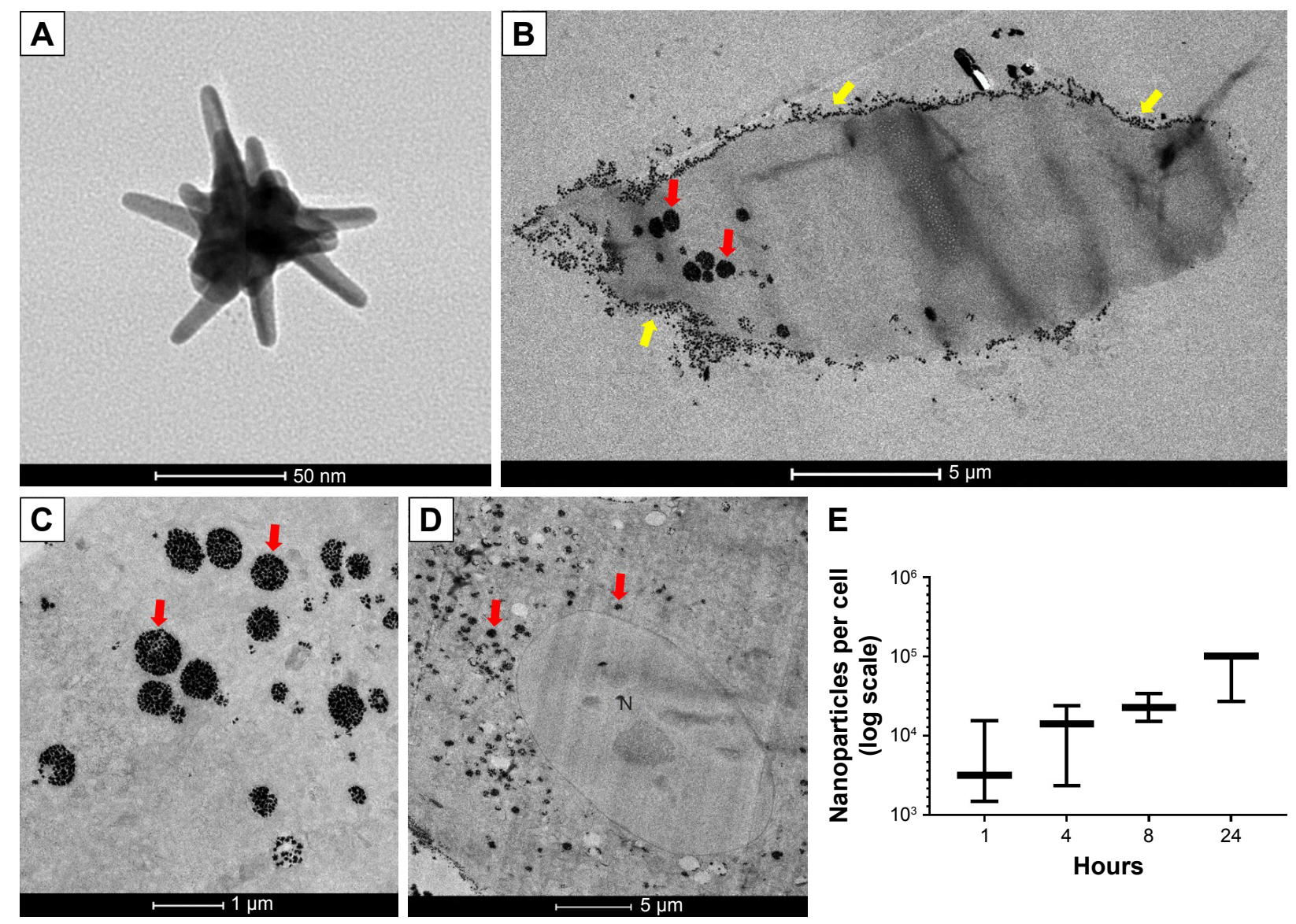

Figure I GNS morphology by TEM (A); visualization and quantification of GNS endocytosed into SUMI49 cell line (B-E). The relative amount of cellular uptake of 0.15 nM GNS after 24-hour incubation was observed by TEM. TEM images show GNS in cytoplasmic vesicles (red arrows) as well as GNS adhered to the cellular membrane (yellow arrows). TEM imaging revealed that the majority of the nanoparticles were localized within the cytoplasmic space and the vesicles of the cells and that no nanoparticles were observed to be present within the nuclear membrane ( $N$, nucleus) of the cell (D). (E) Nanoparticle uptake per cell over a time course of I, 4, 8, and 24 hours of particle incubation with SUMI 49 cell line was quantified by ICP-OES. SUMI 49 cells demonstrated consistent nanoparticle uptake and cytoplasmic retention over a 24 -hour period. Abbreviations: GNS, gold nanostars; TEM, transmission electron microscopy.

\section{GNS exhibit robust endocytosis and vivid TPL in multiple breast cancer cell lines}

In vitro experiments were performed to assess the localization of GNS within the cancer cells and to determine GNS cellular uptake in breast cancer cell subtypes including models of IBC (SUM149 [EGFR activated, estrogen (ER), progesterone, and HER2 negative] and SUM190 [HER2 overexpressed, ER and progesterone receptor negative]) and non-IBC cell lines (BT474M1 [HER2 overexpressed, ER positive] and MDA-MB-231 [triple negative]). Figure 2 shows the cytoplasmic localization of GNS following endocytosis. Concentrated colloidal solutions of GNS appear dark blue in color under phase-contrast imaging and were found to accumulate within the cytoplasm of cells. When viewed under multiphoton microscopy, cells labeled with GNS display intense TPL. These results are in stark contrast to cell lines cultured in media without GNS. Staining with Hoechst 33342 delineates the nucleus to show nanoparticle accumulation within the cytoplasm and outside of the nuclear envelope, and this result is consistent with previous studies. ${ }^{16}$ These results demonstrate that GNS are robustly endocytosed in multiple cancer cell lines, irrespective of receptor expression (Figure 2).

\section{Proliferation and viability of IBC cells are minimally affected by GNS uptake}

Because a high prevalence of SUM149 cell lines obtained from IBC patients are being used in IBC studies, this cell line was further evaluated so as to verify whether this model could be used as a principal model to examine the effects of cytoplasmic GNS accumulation on cell proliferation and viability. The dose- and time-dependent effects of cytoplasmic GNS accumulation on cell proliferation and viability were evaluated using MTT and CellTiter Blue ${ }^{\circledR}$ (resazurin) assays, respectively. Compared to the unlabeled control, increasing intracellular concentrations of GNS did not cause 


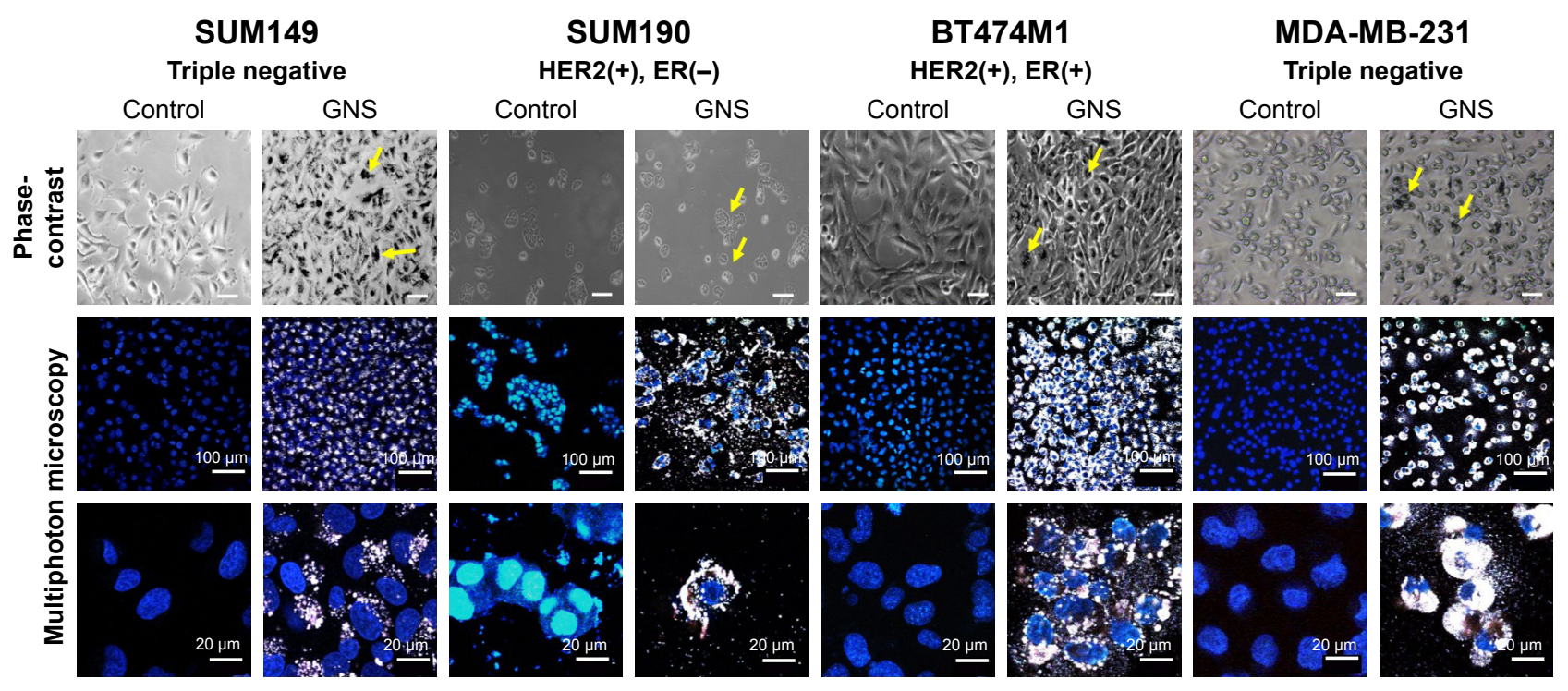

Figure 2 GNS uptake in IBC (SUMI49 \& SUMI90) and non-IBC (BT474MI \& MDA-MB-23I) cell lines. Receptor statuses are provided beneath each represented cell line. The cell lines were incubated with $0.15 \mathrm{nM}$ GNS for 24 hours, washed to remove free particles, stained with Hoechst 33342, and imaged via MPM. The GNS appear as black particles within the cells under phase-contrast imaging (yellow arrows; scale bars $=100 \mu \mathrm{m}$ ). Under MPM, the GNS in the cell cytoplasm appear white in color. Abbreviations: GNS, gold nanostars; MPM, multiphoton microscopy; IBC, inflammatory breast cancer.
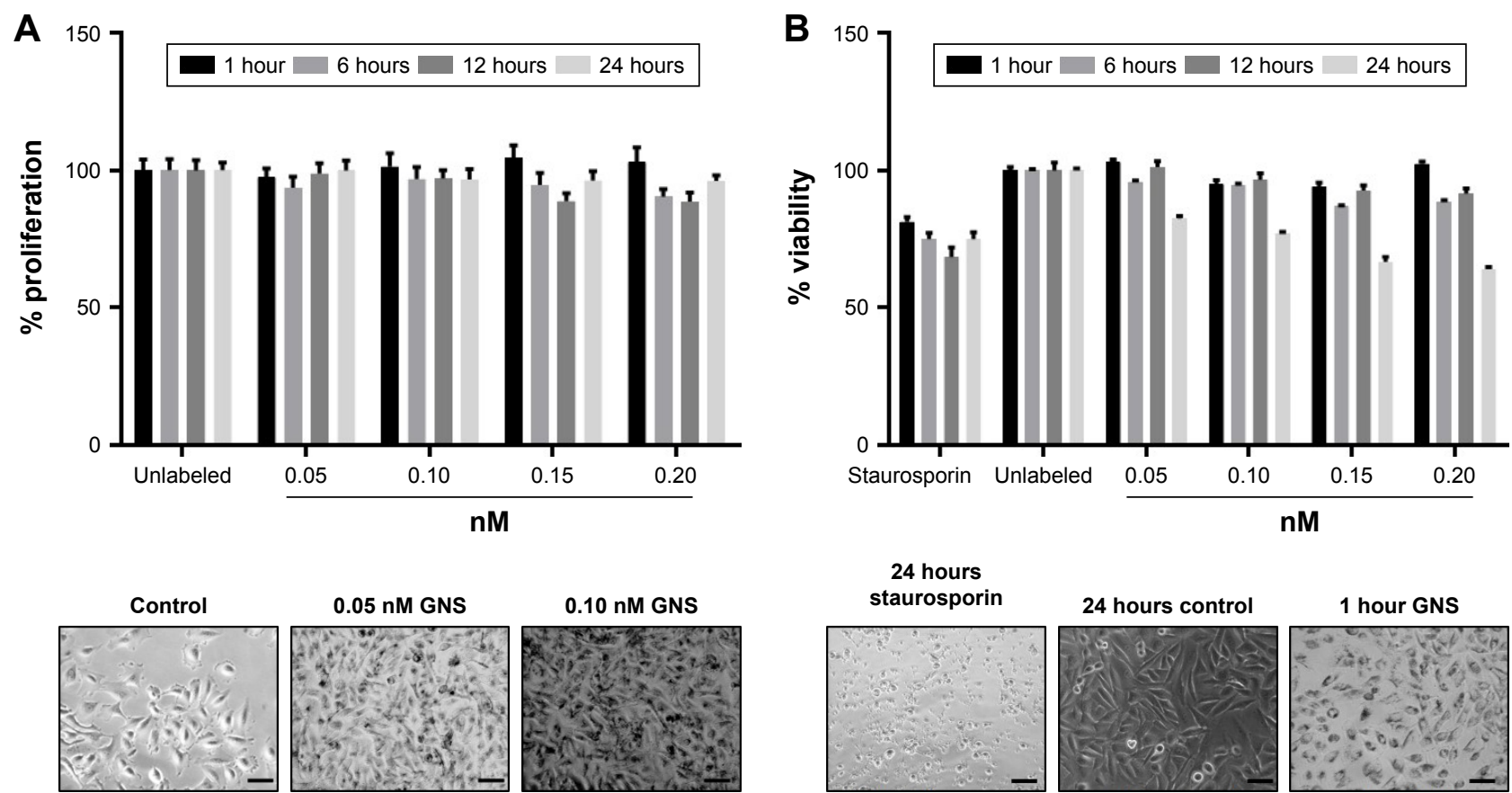

\section{$0.10 \mathrm{nM}$ GNS}

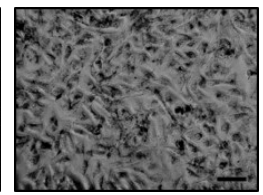

$0.20 \mathrm{nM}$ GNS
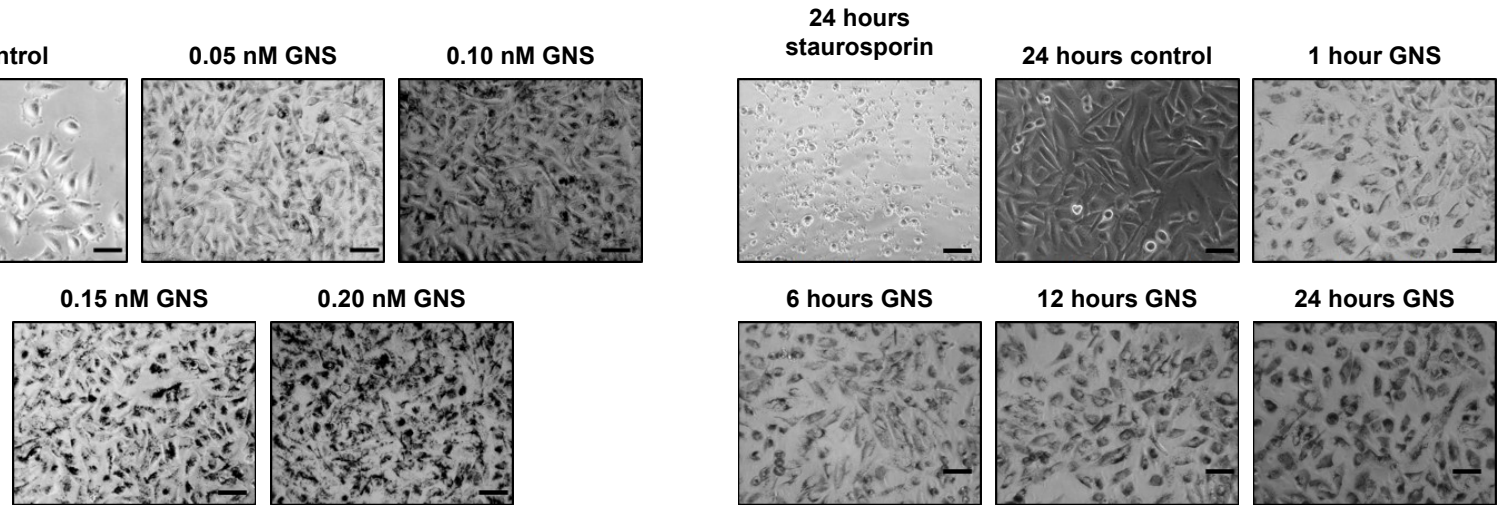

Figure 3 The effects of increasing concentrations of GNS on SUMI49 proliferation and viability following nanoparticle incubation at I, 6, I2, and 24 hours as determined by MTT (A) and resazurin-based assays (B), respectively. SUMI 49 cells were incubated with nanoparticles at concentrations of $0.05,0.10,0.15$, and $0.20 \mathrm{nM}$. After all time points were met, MTT or resazurin assays were performed. Representative phase-contrast images of the experimental groups immediately prior to the addition of MTT or resazurin reagent are shown (scale bars $=100 \mu \mathrm{m}$ ). MTT assays showed minor decreases in cell proliferation at 6, 12 , and 24 hours of GNS incubation, whereas resazurin assays showed a larger decrease in cell viability at 24 hours for all nanoparticle concentrations. The effects of GNS on cell proliferation and viability never precluded the experimental use of GNS-labeled cells.

Abbreviations: GNS, gold nanostars; MTT, (3-(4, 5-dimethylthiazolyl-2)-2, 5-diphenyltetrazolium bromide). 
a noteworthy effect on cellular proliferation (Figure 3A). Interestingly, for all GNS concentrations at 24 hours, there was a slight increase in cellular proliferation compared to other treatment times. This may be due to markedly increased accumulation of GNS in the cell cytoplasm at 24 hours, which resulted in a spectrophotometric interference when the plate reader was used to analyze the results of MTT. Cellular viability assays revealed that for all GNS concentrations, a decrease in cell viability was observed at 24 hours (Figure 3B); however, it should be noted that the incubation of cells with GNS never precluded the experimental use of these cells. These results demonstrate that in the absence of photostimulation, the incubation of cells with GNS has relatively no effect on cellular proliferation and viability.

\section{Efficient cellular ablation of multiple breast cancer cell lines using GNS as a nanotheranostic platform}

In vitro experiments were performed to determine whether GNS cellular ablation differs between IBC (SUM149 and
SUM190) and non-IBC (BT474M1 and MDA-MB-231) cell lines. Following nanoparticle labeling, all GNS-containing cancer cell lines demonstrated efficient photothermal ablation when appropriately stimulated (Figure 4). Live/dead fluorescent staining was used to evaluate cell viability after treatment. Compared to unlabeled control samples exposed to the same laser intensities, GNS-labeled cell lines displayed distinct cellular death. The zone of cellular ablation was confined to the area of laser exposure, demonstrating the specificity and limited off-target effects with nanoparticle cell labeling and photothermal ablation in vitro. These studies demonstrate that GNS allow for efficient photothermal ablation of multiple breast cancer cell lines, irrespective of subtype or hormone receptor expression.

\section{GNS accumulate within IBC tumor emboli, allowing vivid luminescent monitoring in vitro}

After demonstrating the ability to visualize and photothermally ablate monolayers of IBC and non-IBC cell lines,
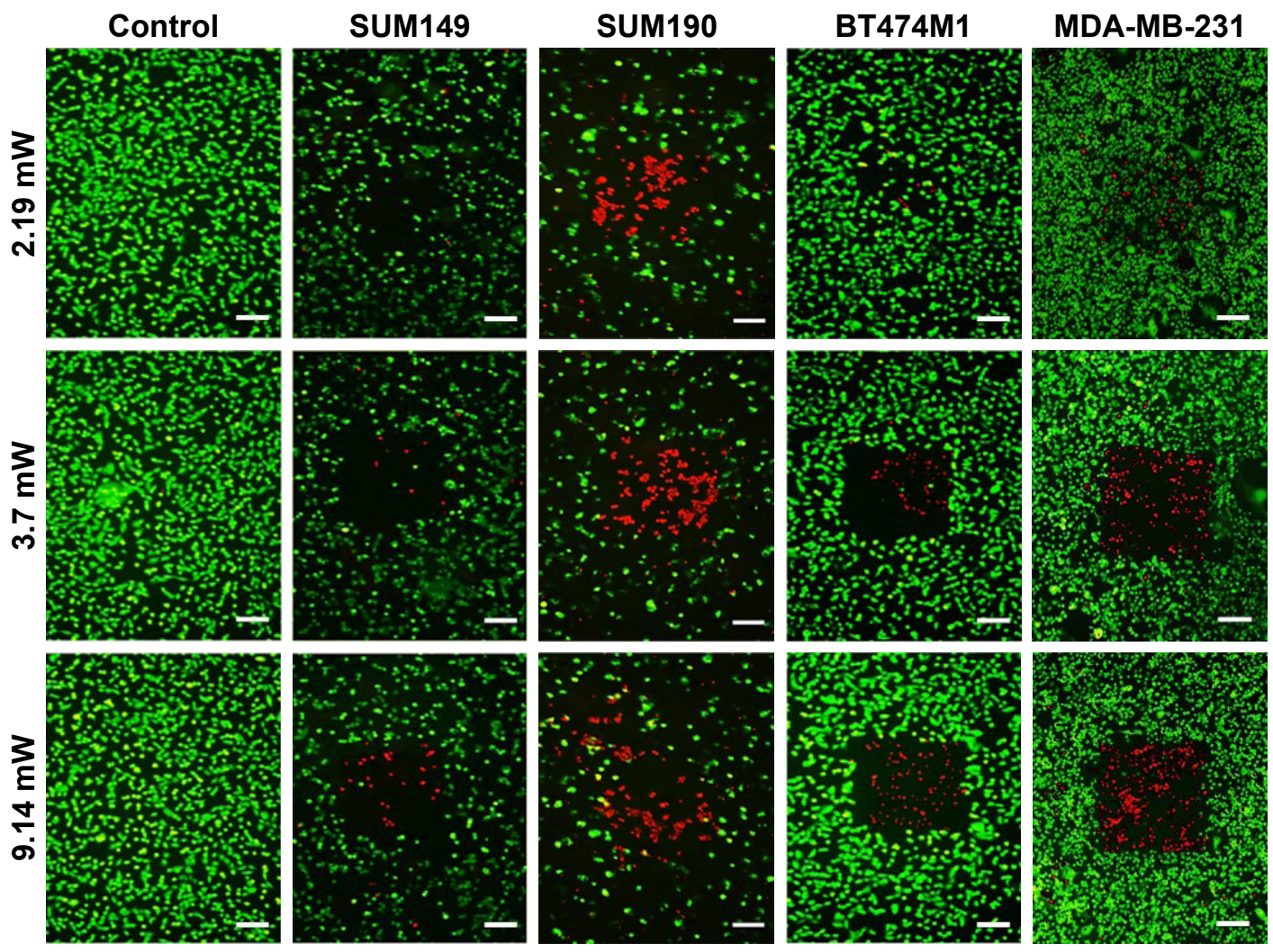

Figure 4 Photothermolysis of IBC (SUMI49 and SUMI90) and non-IBC (BT474MI and MDA-MB-23I) cell lines. Cells were incubated with 0.15 nM GNS for 24 hours, washed to remove free particles, and exposed to an $800 \mathrm{~nm}$ multiphoton pulsed laser for 3 minutes at various laser intensities. Following laser exposure, cells were stained with fluorescein diacetate (live $=$ green) and propidium iodide $($ dead $=$ red) to determine relative cell viability. An empty region of cells is representative of the treatment area where cells detached from the plate's surface. The most effective degree of irradiation was determined to be $3.7 \mathrm{~mW}$, yielding the most specific area of cellular ablation. For all cell lines tested, the control samples (cells without GNS, ie, unlabeled) displayed full viability following photothermal treatment. All samples were plated in 35 mm Petri dishes. Scale bars are $100 \mu \mathrm{m}$.

Abbreviations: GNS, gold nanostars; IBC, inflammatory breast cancer. 
A
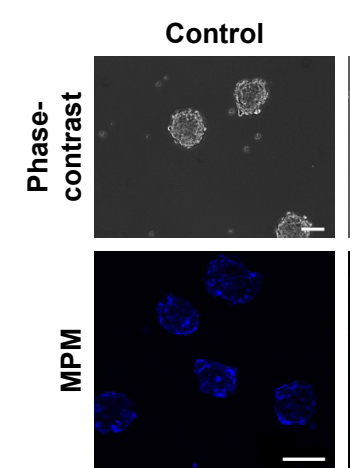

B

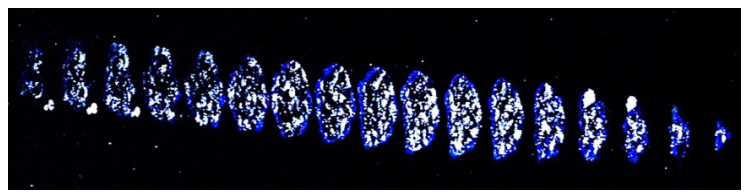

SUM149

GNS
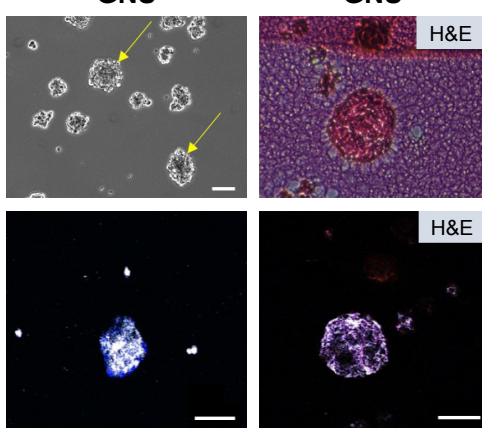

C
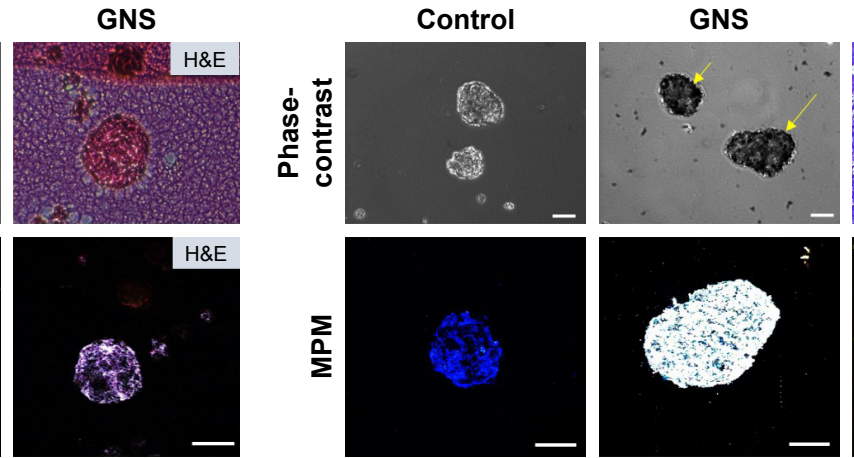

D

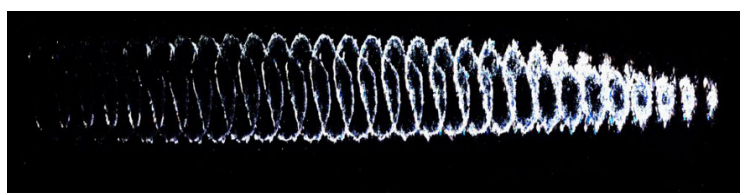

SUM190

GNS

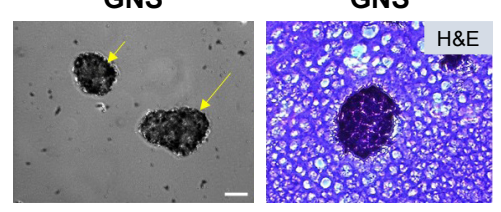

H\&E

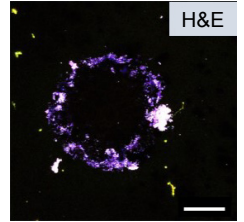

Figure 5 GNS are endocytosed by SUMI49 (A, B) and SUMI90 (C, D) tumor emboli, after which they penetrate into the tumor embolic core. Tumor emboli shown here are imaged after 96 hours of embolic maturation. The control groups represent cells that are free of nanoparticles. For GNS-labeled emboli, nanoparticles were added after 96 hours of embolic maturation. Following 24 hours of GNS incubation, samples were stained with Hoechst 3342 and imaged with MPM. The black portions, as shown by yellow arrows, represent GNS as observed by phase-contrast microscopy. GNS as observed by MPM is shown by white luminescence. H\&E staining displays the crosssectional area of the emboli, and MPM imaging of the cross-section further demonstrates the depth of GNS penetrance into the tumor embolic core (scale bars $=100 \mu \mathrm{m})$. (B, D) 2D projections of the GNS-labeled tumor emboli depicted in $\mathbf{A}$ and $\mathbf{C}$. Shown here are the individual Z slices of the stacked composite image. This projection demonstrates the depth of GNS penetrance into the tumor emboli.

Abbreviations: GNS, gold nanostars; MPM, multiphoton microscopy; H\&E, hematoxylin and eosin.

the feasibility of translating these results to a $3 \mathrm{D}$ tumor emboli culture model was assessed. The results of GNS incubation with tumor emboli are shown in Figure 5. Phasecontrast imaging shows the intra-embolic accumulation of GNS (Figure 5A and C yellow arrows). Under multiphoton microscopy, intense TPL of GNS-labeled tumor emboli can be visualized. Using ImageJ, a 2D projection of individual slices was reconstructed to better delineate the intra-embolic depth of GNS penetrance into the tumor emboli (Figure 5B and D). ${ }^{37}$ As shown in Figure 5B, GNS (which appear white) are homogenously distributed within several layers of the SUM149 tumor emboli, extending from the north to south pole of the embolic structure. In case of SUM190 cell line, which appears to form larger, more compact emboli compared to SUM149, nanoparticle penetrance demonstrated a confinement to the periphery of the emboli (Figure 5D) further confirmed by H\&E staining (Figure $5 \mathrm{~A}$ and $\mathrm{C}$ ). Under phase-contrast imaging, H\&E staining showed $20 \mu \mathrm{m}$ tumor emboli cross-sections after gelatin embedding. These histological samples were also imaged using multiphoton microscopy. The results show an even distribution of GNS over the entire embolic cross-section for SUM149 tumor emboli; however, nanoparticle accumulation in SUM190 tumor emboli was less robust and confined to the structural periphery.

\section{Intracellular accumulation of GNS within IBC tumor emboli allows effective photothermal ablation}

If abundant accumulation of GNS in a homogenous fashion is confirmed within IBC tumor emboli, then the tumor emboli is disrupted and ablated by photothermal treatment. In case of SUM149 and SUM190 tumor emboli, cell death staining with propidium iodide demonstrated that GNSlabeled emboli displayed a substantial degree of cellular death after photothermal treatment compared to unlabeled emboli (Figure 6). Phase-contrast imaging shows that following treatment, the tumor embolic structure was disrupted compared to the controls. Laser power densities of 5 and $9.375 \mathrm{~W} / \mathrm{cm}^{2}$ showed comparable degrees of cellular death following laser treatment. Photothermal treatment with a power density of $2.18 \mathrm{~W} / \mathrm{cm}^{2}$ showed a decrease in the degree of cellular death compared to that with higher laser intensities. Most notably, the area of emboli ablation was confined to the periphery of the tumor emboli compared to higher power densities. Furthermore, it should be noted that photothermal ablation was successfully accomplished in SUM190 cell line, even though this cell line displayed a more peripheral, rather than central, accumulation of GNS (Figure 5D). This suggests that with the use of photothermal ablation, complete penetrance of nanoparticles may not be necessary and that 
A
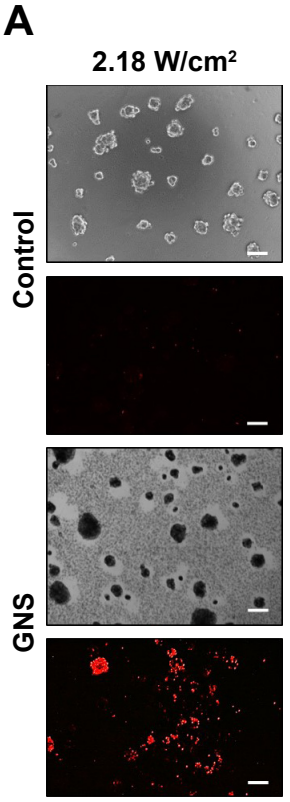

SUM149

$5 \mathrm{~W} / \mathrm{cm}^{2}$
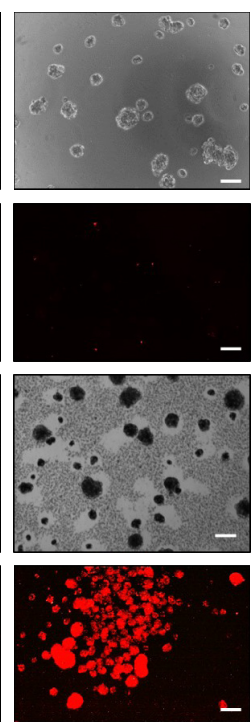
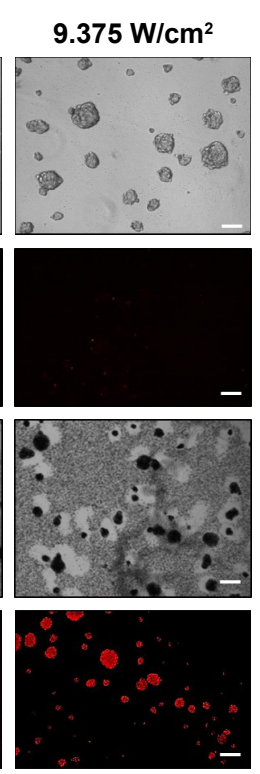

B
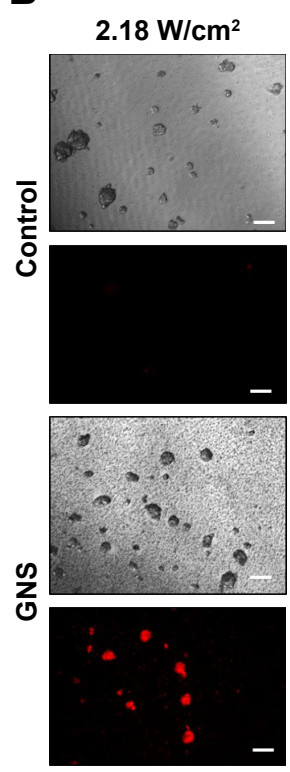

SUM190

$5 \mathrm{~W} / \mathrm{cm}^{2}$
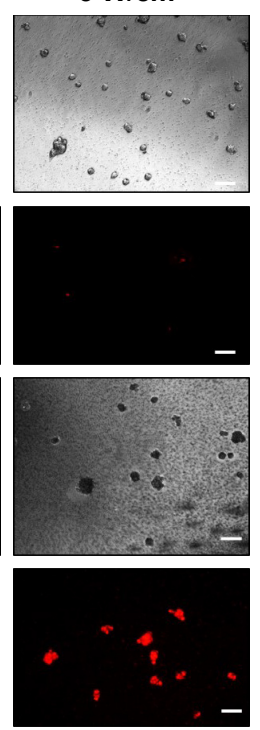

$9.375 \mathrm{~W} / \mathrm{cm}^{2}$
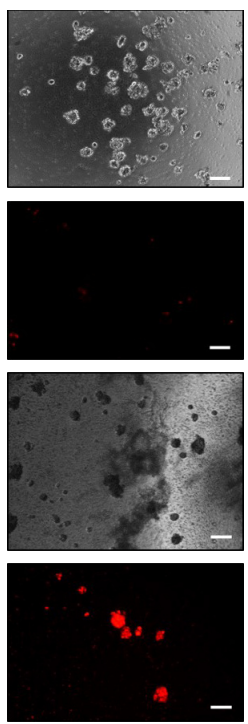

Figure 6 Photothermal treatment of SUMI49 (A) and SUMI90 (B) tumor emboli. After 96 hours of tumor emboli maturation, 0.15 nM of GNS was incubated with tumor emboli for 24 hours. Following GNS incubation, samples were exposed to an $808 \mathrm{~nm}$ continuous laser at various laser intensities. After treatment, the experimental samples were stained with propidium iodide to illustrate any cellular death that occurred following laser irradiation. All emboli that were incubated with GNS exhibited cell death following laser exposure. Control samples were treated with the same level of irradiation, which demonstrated virtually no cell death. Representative phase-contrast images of each sample following treatment are shown. Empty spaces seen under phase-contrast for GNS-labeled emboli are due to the repositioning of the emboli in the wells following photothermal treatment. The GNS-labeled emboli appear in black color under phase-contrast imaging due to GNS uptake, compared to the unlabeled controls (scale bars $=100 \mu \mathrm{m}$ ).

Abbreviation: GNS, gold nanostars.

a peripheral accumulation could be sufficient to disrupt and ablate tumor emboli.

\section{Efficient nanoparticle uptake and photothermal ablation are achieved in drug-resistant IBC cell line}

After confirming that nanoparticles are readily endocytosed and possess the ability to photothermally ablate IBC cells in both $2 \mathrm{D}$ and $3 \mathrm{D}$ culture models, the next step was to investigate the use of GNS in drug-resistant IBC cell line rSUM149. It has been shown that nanocarriers designed to treat drugsensitive cell lines may not be effective in drug-resistant cell lines because of the differing lipid composition and consequential biophysical characteristics. Lipids play a major role in the regulation of membrane trafficking and endocytosis, the primary mechanism of uptake of nanoparticle-based systems. ${ }^{39}$ Vijayaraghavalu et al investigated the use of Doxil ${ }^{\circledR}$, the liposomal formulation of doxorubicin, in delivering doxorubicin to resistant breast cancer cells. ${ }^{40}$ The compact and rigid nature of drug-resistant cell membranes resulted in impaired endocytic function that inhibited intracellular drug delivery using Doxil. Although the study was carried out using the encapsulated formulation Doxil, issues of endocytic transport in drug-resistant cells could potentially exist with other PEGylated nanocarrier systems, such as the nanoparticles used in this study. In vitro experiments were performed to assess the localization of GNS within the rSUM149 cell line, a model that was generated from SUM149 by clonal selection of cell death-resistant population of cells when chronically exposed to EGFR dual kinase inhibitor (lapatinib). rSUM149 cell lines were subsequently identified to acquire resistance to various EGFR targeting drugs, chemotherapy and immunotherapeutic modulation due to increased expression of anti-apoptotic and antioxidant proteins that increase tumor cell survival signaling. ${ }^{36,41,42}$ As shown in Figure 7A, GNS are readily endocytosed into the cytoplasm of the rSUM149 cell line and result in successful photothermal ablation (Figure 7B). Similar to the SUM149 and SUM190 cell lines, our results revealed that GNS are distributed within multiple layers of the rSUM149 tumor emboli (Figure 7C). H\&E stained cross-sections under phasecontrast imaging and MPM showed a distribution of GNS over the entire embolic cross-section for rSUM149 cell lines (Figure 7C). Finally, cellular death staining of the rSUM149 tumor emboli following photothermal treatment with NIR laser demonstrated efficient photothermal ablation and emboli disruption, which is similar to those results obtained for SUM149 and SUM190 IBC cell lines (Figure 7D). 


\section{A}
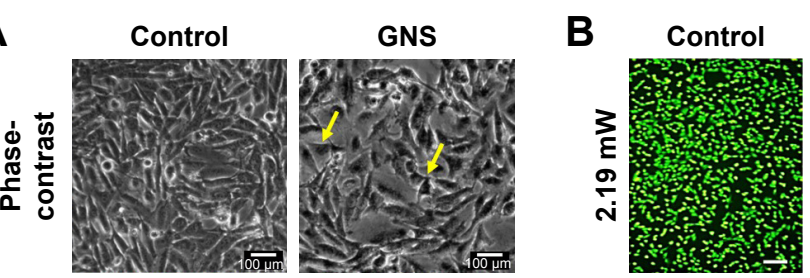

GNS
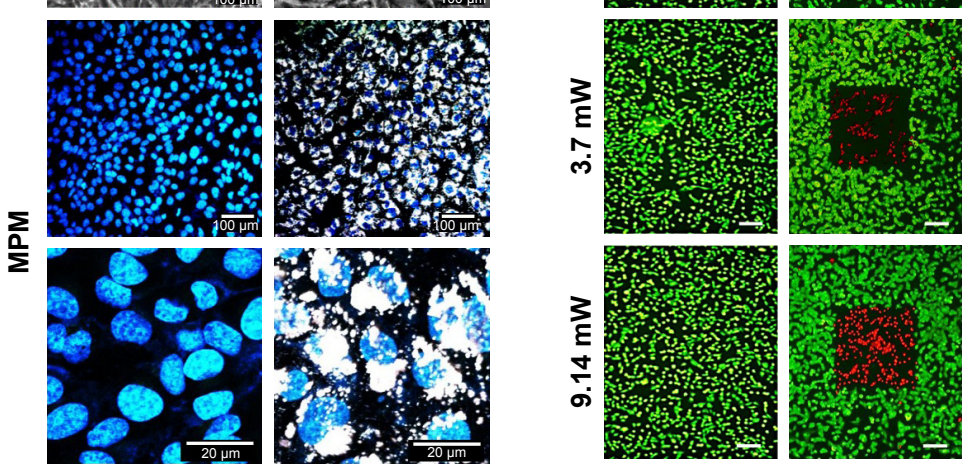

C
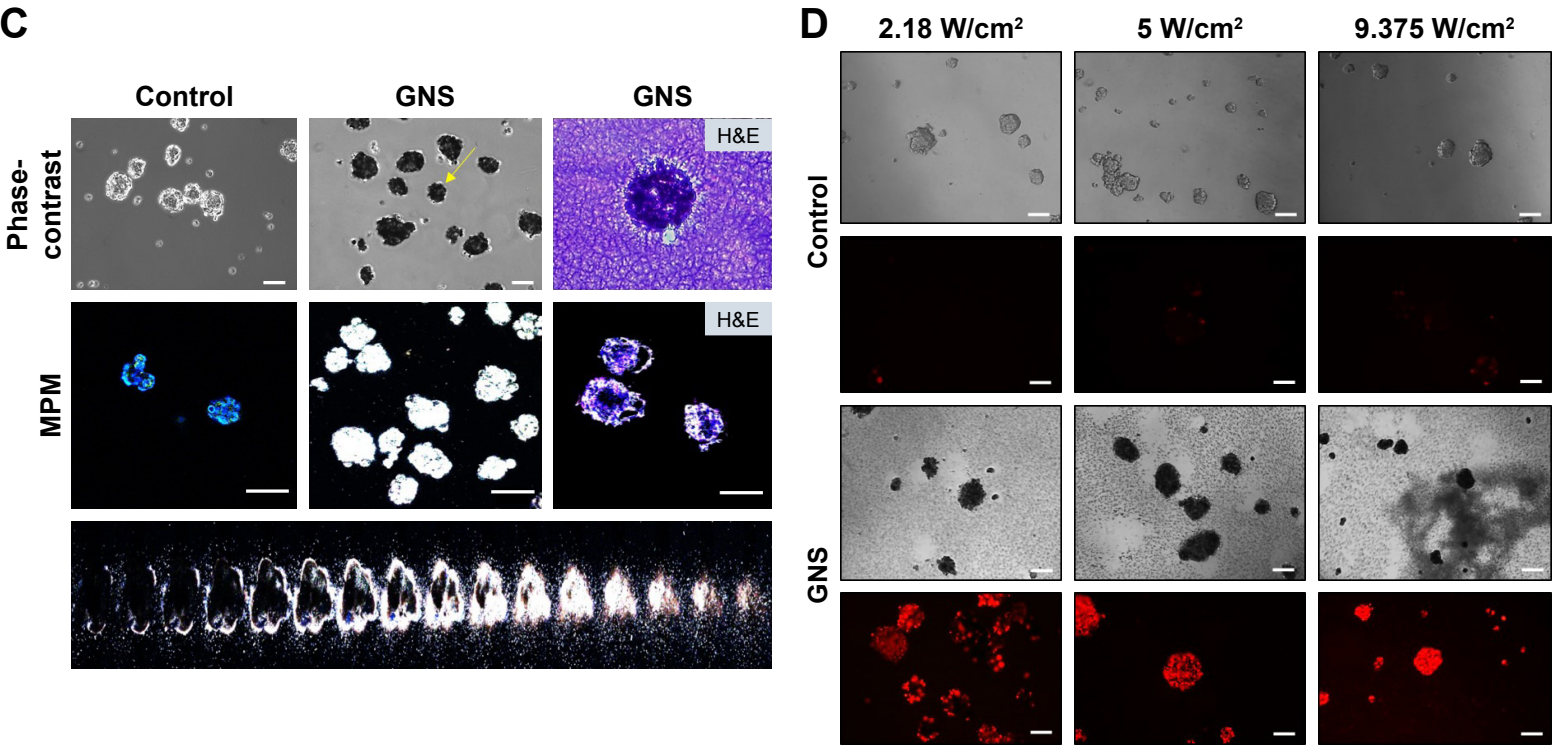

Figure 7 GNS uptake and photothermal ablation in drug-resistant IBC cell line rSUMI 49. (A) The drug-resistant IBC variant, rSUMI49, was incubated with 0.15 nM GNS for 24 hours, stained with Hoechst 33342 and imaged by MPM. The GNS in the cells appear as black particles as observed by phase-contrast imaging (yellow arrows), whereas the GNS in the cytoplasm appear as white luminiscence under MPM. (B) Photothermolysis of rSUMI 49 cell line. Cells were incubated with 0.15 nM GNS for 24 hours and then exposed to a multiphoton pulsed laser for 3 minutes at various laser intensities. Following laser exposure, cells were stained with fluorescein diacetate (live $=$ green) and propidium iodide $(\mathrm{dead}=\mathrm{red})$ to determine relative cell viability. The control samples (cells without GNS, ie, unlabeled) displayed full viability following photothermal treatment (scale bars $=100 \mu \mathrm{m}$ ). (C) GNS penetrate rSUMI49 tumor emboli. Tumor emboli shown here are imaged after 96 hours of embolic maturation. The control groups represent cells that are free of nanoparticle. For GNS-labeled emboli, nanoparticles were added after 96 hours of embolic maturation. Following 24 hours of GNS incubation, samples were stained with Hoechst 3342 and imaged with MPM. The black portions, shown by yellow arrows, represent GNS as observed by phase-contrast microscopy, whereas white luminescence represents GNS as observed by MPM (scale bars $=100 \mu \mathrm{m}$ ). A 2D projection of the GNS-labeled tumor emboli is shown where individual $Z$ slices of the stacked composite image are displayed. This projection demonstrates the depth of GNS penetrance into the tumor emboli. H\&E staining displays the cross-sectional area of the emboli; MPM imaging of the cross-section further demonstrates the depth of GNS penetrance into the tumor embolic core. (D) Photothermal treatment of rSUMI49 tumor emboli. Following GNS incubation, samples were exposed to an $808 \mathrm{~nm}$ continuous laser at various laser intensities. After treatment, the experimental samples were stained with propidium iodide to illustrate any cellular death that occurred following laser irradiation. All emboli that were incubated with GNS exhibited cell death following laser exposure. Control samples were treated with the same level of irradiation, which demonstrated virtually no cell death. Representative phase-contrast images of each sample are shown following treatment. Empty spaces seen under phase-contrast imaging for GNS-labeled emboli are due to the repositioning of the emboli following photothermal treatment. The GNS-labeled emboli appear in black color under phase-contrast imaging due to GNS uptake, compared to the unlabeled controls (scale bars $=100 \mu \mathrm{m}$ ).

Abbreviations: GNS, gold nanostars; H\&E, hematoxylin and eosin; MPM, multiphoton microscopy.

\section{Discussion}

Despite improved screening and advanced treatment modalities to reduce the incidence and mortality of breast cancer, significant challenges remain in developing effective therapies with limited adverse effects and off-target toxicity. ${ }^{43}$ IBC is a form of locally advanced breast cancer that is characterized by rapid onset of symptoms and accelerated growth due to the development of lymphovascular emboli. ${ }^{6}$ 
An obstacle to advancing the study of nanotherapeutics for IBC is the difficulty associated with mimicking the physical conditions found in the dermal lymphatics to more accurately recapitulate a clinical model of IBC tumor emboli. In this study, we have used a biologically relevant in vitro preclinical model to study the feasibility of a visualization and photothermal treatment technique for IBC using plasmonic GNS as nanotheranostic platform.

Often, a single treatment modality, such as chemotherapy, will not produce a complete therapeutic response. Using combination therapies has been shown to increase the response rate and effectiveness of treatment. ${ }^{44}$ One modality that has gained increasing interest is hyperthermic therapy, which means an increase in tissue temperature at a range of $40^{\circ} \mathrm{C}-44^{\circ} \mathrm{C}$, and it has been shown to help improve local control in patients with advanced breast cancer. ${ }^{45,46}$ However, adverse effects (ie, pain, bleeding, infection, and skin burns) continue to be a limitation to the widespread adoption of hyperthermic therapy, partially due to nonspecific heating of tissue by currently available hyperthermic treatments that utilize radio waves, microwaves, or laser energy. ${ }^{47}$ Furthermore, current hyperthermic treatments are highly invasive as they are dependent on the insertion of a probe into the lesion site. Utilization of metallic nanoparticles, such as GNS, that preferentially accumulate in malignant tissue through the EPR effect limits the off-target systemic accumulation of these particles. ${ }^{20}$ Additionally, the ability of GNS to convert electromagnetic energy into heat energy makes it a potential candidate to be used in targeted and site-specific hyperthermic therapy with decreased laser power. The use of metallic nanoparticles also eliminates the need for invasive probes in hyperthermic therapy and instead allows for noninvasive thermal heating by using NIR lasers.

Photothermal therapy with a NIR laser has been shown to be an effective mode of treatment for breast cancer cell lines in vitro. ${ }^{48-50}$ Previous studies have focused on the use of gold nano-raspberries, nanocages, and nanostars for the photothermal ablation of SKBR3 breast cancer cells in 2D monoculture. ${ }^{16,51,52}$ Another study looked at the effects of GNS-mediated photothermal therapy using a NIR laser in BT549 breast cancer cells and found that photothermal ablation could be accomplished using ultra-low irradiance below the maximal permissible exposure of skin. ${ }^{16}$ However, very few studies have examined the use of nanoparticle-mediated photothermal therapy in the aggressive subtype of locally advanced breast cancer, IBC. Our results demonstrate that effective photothermal ablation is accomplished in multiple breast cancer cell lines, including IBC tumor cells, irrespective of differences in cell receptor status or drug resistance.
Majority of the in vitro studies have examined the use of nanoparticle therapeutics in traditional 2D in vitro cultures. Therefore, only the study of direct cellular interactions with nanoparticles was possible; however, it did not offer the ability to explore the interactions of nanoparticles with tissue structures in a controlled manner. Furthermore, in a 3D culture model, physical cell-cell interactions and extracellular matrices are altered, which may affect nanoparticle uptake and delivery. ${ }^{25,26,53}$ This study examined the use of nanoparticles in a 3D model of IBC cell lines and showed that differences in uptake patterns do indeed exist with respect to nanoparticle endocytosis in $2 \mathrm{D}$ versus $3 \mathrm{D}$ culture. In all 2D cultures, nanoparticle endocytosis was equivocally robust and led to efficient photothermal ablation using a pulsed laser. Furthermore, TEM imaging showed that the majority of nanoparticles were found to be housed within both the cytoplasmic space and the vesicles of cells, consistent with previous studies. ${ }^{20}$ This compartmentalization can be attributed to the relatively large size of GNS (50 nm), which precludes their entry into the nuclear membrane pores. ${ }^{54,55}$ In addition, ICP-OES analysis revealed consistent nanoparticle uptake and cellular retention across cell lines over a 24-hour period. When transitioned to a $3 \mathrm{D}$ tumor emboli model, nanoparticle endocytosis was confined to the periphery of the larger SUM190 tumor emboli compared to its SUM149 and rSUM149 counterparts. In addition, the effects of photothermal ablation were less efficacious in the $3 \mathrm{D}$ tumor emboli compared to 2D cultures, with the majority of cellular death confined to the embolic periphery at lower laser intensities; in the 2D cell cultures, the efficacy of photothermal ablation was nearly equivalent across all laser intensities studied.

The use of nanotherapeutics is not limited only to photothermal therapy. Ideally, it would extend to the synergistic use of selective photothermolysis with drug delivery. ${ }^{53,56}$ This aids in overcoming any potential hyperthermic resistance that may exist in various cancer subtypes. ${ }^{57-59}$ However, to effectively utilize this combination therapy, the nanoparticles need to successfully penetrate into the core of the tumor embolic structures that exist in IBC. This study found that for SUM149 and rSUM149 cell lines, nanoparticles were homogenously distributed throughout the tumor embolic structure, as confirmed by MPM and histopathological staining. Even though GNS accumulation was largely confined to the periphery of the larger SUM190 tumor emboli, effective photothermal ablation was still achieved. This suggests that for photothermal therapy, spatially distributed heat derived from the GNS is sufficient for tumor cell ablation and, therefore, complete nanoparticle penetrance may not 
be necessary. While these studies are promising and suggest a potential role of nanoparticles in drug delivery to IBC tumor emboli, future studies utilizing nanoparticle drug delivery in conjunction with photothermal therapy should be conducted in a $3 \mathrm{D}$ model to assess the relative penetrance needed for effective synergistic therapy.

\section{Conclusion}

In summary, use of $2 \mathrm{D}$ cell culture is a rapid way of screening potential therapies, including nanotherapeutics. However, these cultures do not accurately represent the cell-cell interactions that occur in vivo. A good bridging point between in vitro and in vivo studies is the use of $3 \mathrm{D}$ tumor spheroid and emboli cultures that better mimic the in vivo tumor microenvironments. This is the first study to examine the use of nanoparticles in combination with tumor emboli and to report the effective photothermal ablation of IBC cells in a 3D tumor emboli model. These results will pave way for future studies to further investigate the uptake patterns of nanoparticles in various IBC emboli and also to explore how these patterns may be influenced by tumor microenvironments. Furthermore, these results provide a robust step to further evaluate the effectiveness of synergistic photothermal therapy and drug delivery in a preclinical model of IBC.

\section{Abbreviations}

NIR, near infrared; ER, estrogen; EGFR, epidermal growth factor receptor; MTT, (3-(4, 5-dimethylthiazolyl-2)-2, 5-diphenyltetrazolium bromide); GNS, gold nanostars; EPR, enhanced permeability and retention; PEG, polyethylene glycol; TPL, two-photon luminescence; TAT, transactivator of transcription; IBC, inflammatory breast cancer; ICP-OES, inductively coupled optical emission spectrometry; TEM, transmission electron microscopy; H\&E, hematoxylin and eosin; MPM, multiphoton microscopy; 2D, two dimensional; 3D, three dimensional.

\section{Acknowledgments}

The authors would like to thank members of Devi Lab, Pranalee Patel and Scott Sauer, for assistance in cell culture models; and Kim Hutchison and the North Carolina State University Analytical Spectroscopy Service Laboratory for their assistance with ICP-OES analysis.

This work was supported in part by pilot research funds from the Duke Cancer Institute (as part of the P30CA014236) to GRD, Department of Surgery Clarence Gardner Award (GRD), School of Medicine Bridge and Duke Consortium for
Inflammatory Breast Cancer funds (GRD) and Duke Faculty Exploratory Funding (TD). This study was based upon the work supported by the National Science Foundation Graduate Research Fellowship under Grant No 1106401 (BMC).

\section{Disclosure}

The authors report no conflicts of interest in this work.

\section{References}

1. Kleer CG, van Golen KL, Merajver SD. Molecular biology of breast cancer metastasis. Inflammatory breast cancer: clinical syndrome and molecular determinants. Breast Cancer Res. 2000;2(6):423-429.

2. Woodward WA. Inflammatory breast cancer: unique biological and therapeutic considerations. Lancet Oncol. 2015;16(15):e568-e576.

3. Matro JM, Li T, Cristofanilli M, et al. Inflammatory breast cancer management in the national comprehensive cancer network: the disease, recurrence pattern, and outcome. Clin Breast Cancer. 2015; 15(1):1-7.

4. Cristofanilli M, Valero V, Buzdar AU, et al. Inflammatory breast cancer (IBC) and patterns of recurrence: understanding the biology of a unique disease. Cancer. 2007;110(7):1436-1444.

5. Robertson FM, Bondy M, Yang W, et al. Inflammatory breast cancer: the disease, the biology, the treatment. CA Cancer J Clin. 2010;60(6): 351-375.

6. Vermeulen PB, van Golen KL, Dirix LY. Angiogenesis, lymphangiogenesis, growth pattern, and tumor emboli in inflammatory breast cancer. Cancer. 2010;116(11 Suppl):2748-2754.

7. Arora J, Sauer SJ, Tarpley M, et al. Inflammatory breast cancer tumor emboli express high levels of anti-apoptotic proteins: use of a quantitative high content and high-throughput 3D IBC spheroid assay to identify targeting strategies. Oncotarget. 2017;8(16):25848-25863.

8. Woodward WA, Cristofanilli M. Inflammatory breast cancer. Semin Radiat Oncol. 2009;19(4):256-265.

9. Nath S, Devi GR. Three-dimensional culture systems in cancer research: focus on tumor spheroid model. Pharmacol Ther. 2016;163:94-108.

10. Ruoslahti E, Bhatia SN, Sailor MJ. Targeting of drugs and nanoparticles to tumors. J Cell Biol. 2010;188(6):759.

11. Kolhatkar R, Lote A, Khambati H. Active tumor targeting of nanomaterials using folic acid, transferrin and integrin receptors. Curr Drug Discov Technol. 2011;8(3):197-206.

12. Gao N, Chen Y, Li L, et al. Shape-dependent two-photon photoluminescence of single gold nanoparticles. J Phys Chem C. 2014;118(25): 13904-13911.

13. Shammas RL, Fales AM, Crawford BM, et al. Human adipose-derived stem cells labeled with plasmonic gold nanostars for cellular tracking and photothermal cancer cell ablation. Plast Reconstr Surg. 2017;139(4): 900e-910e.

14. Yuan H, Khoury CG, Hwang H, Wilson CM, Grant GA, Vo-Dinh T. Gold nanostars: surfactant-free synthesis, 3D modelling, and two-photon photoluminescence imaging. Nanotechnology. 2012;23(7):075102.

15. Niu W, Chua YAA, Zhang W, Huang H, Lu X. Highly symmetric gold nanostars: crystallographic control and surfaceenhanced Raman scattering property. J Am Chem Soc. 2015;137(33):10460-10463.

16. Yuan H, Fales AM, Vo-Dinh T. TAT peptide-functionalized gold nanostars: enhanced intracellular delivery and efficient NIR photothermal therapy using ultralow irradiance. J Am Chem Soc. 2012;134(28): 11358-11361.

17. Kolja T, Salamon J, Szwargulski P, et al. Increasing the sensitivity for stem cell monitoring in system-function based magnetic particle imaging. Phys Med Biol. 2016;61(9):3279-3290.

18. Ariza de Schellenberger A, Kratz H, Farr TD, et al. Labeling of mesenchymal stem cells for MRI with single-cell sensitivity. Int $J$ Nanomedicine. 2016;11:1517-1535. 
19. Liu S, Tay LM, Anggara R, et al. Long-term tracking mesenchymal stem cell differentiation with photostable fluorescent nanoparticles. ACS Appl Mater Interfaces. 2016;8(19):11925-11933.

20. Liu Y, Yuan H, Fales AM, Register JK, Vo-Dinh T. Multifunctional gold nanostars for molecular imaging and cancer therapy. Front Chem. 2015;3:51.

21. Petros RA, DeSimone JM. Strategies in the design of nanoparticles for therapeutic applications. Nat Rev Drug Discov. 2010;9(8):615-627.

22. Vinogradov SV, Bronich TK, Kabanov AV. Nanosized cationic hydrogels for drug delivery: preparation, properties and interactions with cells. Adv Drug Deliv Rev. 2002;54(1):135-147.

23. Whitehead KA, Matthews J, Chang PH, Niroui F, Dorkin JR, Severgnini M, Anderson DG. In vitro-in vivo translation of lipid nanoparticles for hepatocellular siRNA delivery. ACS nano. 2012;6(8): 6922-6929.

24. Arvizo R, Bhattacharya R, Mukherjee P. Gold nanoparticles: opportunities and challenges in nanomedicine. Expert Opin Drug Deliv. 2010; 7(6):753-763.

25. Lee J, Lilly GD, Doty RC, Podsiadlo P, Kotov NA. In vitro toxicity testing of nanoparticles in 3D cell culture. Small. 2009;5(10): 1213-1221.

26. Sambale F, Lavrentieva A, Stahl F, et al. Three dimensional spheroid cell culture for nanoparticle safety testing. J Biotechnol. 2015;205: 120-129.

27. Edmondson R, Broglie JJ, Adcock AF, et al. Three-dimensional cell culture systems and their applications in drug discovery and cell-based biosensors. Assay Drug Dev Technol. 2014;12(4):207-218.

28. Lovitt CJ, Shelper TB, Avery VM. Advanced cell culture techniques for cancer drug discovery. Biology (Basel). 2014;3(2):345-367.

29. Imamura Y, Mukohara T, Shimono Y, et al. Comparison of 2D- and $3 \mathrm{D}$-culture models as drug-testing platforms in breast cancer. Oncol Rep. 2015;33(4):1837-1843.

30. Nyga A, Cheema U, Loizidou M. 3D tumour models: novel in vitro approaches to cancer studies. J Cell Commun Signal. 2011;5(3): 239-248.

31. Mehta G, Hsiao AY, Ingram M, Luker GD, Takayama S. Opportunities and challenges for use of tumor spheroids as models to test drug delivery and efficacy. J Control Release. 2012;164(2):192-204.

32. Dam DHM, Lee JH, Sisco PN, et al. Direct observation of nanoparticle - cancer cell nucleus interactions. ACS Nano. 2012;6(4): 3318-3326.

33. Barbosa S, Topete A, Alatorre-Meda M, et al. Targeted combinatorial therapy using gold nanostars as theranostic platforms. J Phys Chem C. 2014;118(45):26313-26323.

34. Aird KM, Ding X, Baras A, et al. Trastuzumab signaling in ErbB2-overexpressing inflammatory breast cancer correlates with X-linked inhibitor of apoptosis protein expression. Mol Cancer Ther. 2008;7(1):38-47.

35. Williams KP, Allensworth JL, Ingram SM, et al. Quantitative high-throughput efficacy profiling of approved oncology drugs in inflammatory breast cancer models of acquired drug resistance and re-sensitization. Cancer Lett. 2013;337(1):77-89.

36. Aird KM, Ghanayem RB, Peplinski S, Lyerly HK, Devi GR. X-linked inhibitor of apoptosis protein inhibits apoptosis in inflammatory breast cancer cells with acquired resistance to an ErbB1/2 tyrosine kinase inhibitor. Mol Cancer Ther. 2010;9(5):1432-1442.

37. Schneider CA, Rasband WS, Eliceiri KW. NIH Image to ImageJ: 25 years of image analysis. Nat Methods. 2012;9(7):671-675.

38. Brown DA, Chou YF, Beygui RE, Dunn JC, Wu BM. Gelatin-embedded cell-polymer constructs for histological cryosectioning. J Biomed Mater Res B Appl Biomater. 2005;72(1):79-85.

39. Peetla C, Vijayaraghavalu S, Labhasetwar V. Biophysics of cell membrane lipids in cancer drug resistance: implications for drug transport and drug delivery with nanoparticles. Adv Drug Deliv Rev. 2013;65(13-14): 1686-1698.
40. Vijayaraghavalu S, Peetla C, Lu S, Labhasetwar V. Epigenetic modulation of the biophysical properties of drug-resistant cell lipids to restore drug transport and endocytic functions. Mol Pharm. 2012;9(9): 2730-2742.

41. Nair S, Aldrich AJ, McDonnell E, et al. Immunologic targeting of FOXP3 in inflammatory breast cancer cells. PLoS One. 2013;8(1): e53150.

42. Evans MK, Sauer SJ, Nath S, Robinson TJ, Morse MA, Devi GR. $\mathrm{X}$-linked inhibitor of apoptosis protein mediates tumor cell resistance to antibody-dependent cellular cytotoxicity. Cell Death Dis. 2016;7: e2073.

43. DeSantis C, Siegel R, Bandi P, Jemal A. Breast cancer statistics, 2011. CA Cancer J Clin. 2011;61(6):409-418.

44. Newman EA, Guest AB, Helvie MA, et al. Changes in surgical management resulting from case review at a breast cancer multidisciplinary tumor board. Cancer. 2006;107(10):2346-2351.

45. Jones EL, Oleson JR, Prosnitz LR, et al. Randomized trial of hyperthermia and radiation for superficial tumors. J Clin Oncol. 2005; 23(13):3079-3085.

46. Varma S, Myerson R, Moros E, Taylor M, Straube W, Zoberi I. Simultaneous radiotherapy and superficial hyperthermia for high-risk breast carcinoma: a randomised comparison of treatment sequelae in heated versus non-heated sectors of the chest wall hyperthermia. Int J Hyperthermia. 2012;28(7):583-590.

47. Lee J, Chatterjee DK, Lee MH, Krishnan S. Gold nanoparticles in breast cancer treatment: promise and potential pitfalls. Cancer Lett. 2014; 347(1):46-53.

48. Hirsch LR, Stafford RJ, Bankson JA, et al. Nanoshell-mediated nearinfrared thermal therapy of tumors under magnetic resonance guidance. Proc Natl Acad Sci U S A. 2003;100(23):13549-13554.

49. Loo C, Lowery A, Halas N, West J, Drezek R. Immunotargeted nanoshells for integrated cancer imaging and therapy. Nano Lett. 2005;5(4): 709-711.

50. Lowery AR, Gobin AM, Day ES, Halas NJ, West JL. Immunonanoshells for targeted photothermal ablation of tumor cells. Int J Nanomedicine. 2006;1(2):149-154.

51. Gandra N, Portz C, Nergiz SZ, Fales A, Vo-Dinh T, Singamaneni S. Inherently stealthy and highly tumor-selective gold nanoraspberries for photothermal cancer therapy. Sci Rep. 2015;5:10311.

52. Skrabalak SE, Chen J, Au L, Lu X. Gold nanocages for biomedical applications. Adv Mater. 2007;19(20):3177-3184.

53. Abadeer NS, Murphy CJ. Recent progress in cancer thermal therapy using gold nanoparticles. J Phys Chem C. 2016;120(9):4691-4716.

54. Oh E, Delehanty JB, Sapsford KE, et al. Cellular uptake and fate of PEGylated gold nanoparticles is dependent on both cell-penetration peptides and particle size. ACS Nano. 2011;5(8):6434-6448.

55. Huang K, Ma H, Liu J, et al. Size-dependent localization and penetration of ultrasmall gold nanoparticles in cancer cells, multicellular spheroids, and tumors in vivo. ACS Nano. 2012;6(5):4483-4493.

56. Wust P, Hildebrandt B, Sreenivasa G, et al. Hyperthermia in combined treatment of cancer. Lancet Oncol. 2002;3(8):487-497.

57. Huang HC, Yang Y, Nanda A, Koria P, Rege K. Synergistic administration of photothermal therapy and chemotherapy to cancer cells using polypeptide-based degradable plasmonic matrices. Nanomedicine (Lond). 2011;6(3):459-473.

58. Rylander MN, Feng Y, Bass J, Diller KR. Thermally induced injury and heat-shock protein expression in cells and tissues. Ann N Y Acad Sci. 2005;1066:222-242.

59. Burke AR, Singh RN, Carroll DL, et al. The resistance of breast cancer stem cells to conventional hyperthermia and their sensitivity to nanoparticle-mediated photothermal therapy. Biomaterials. 2012;33(10): 2961-2970. 


\section{Supplementary material}

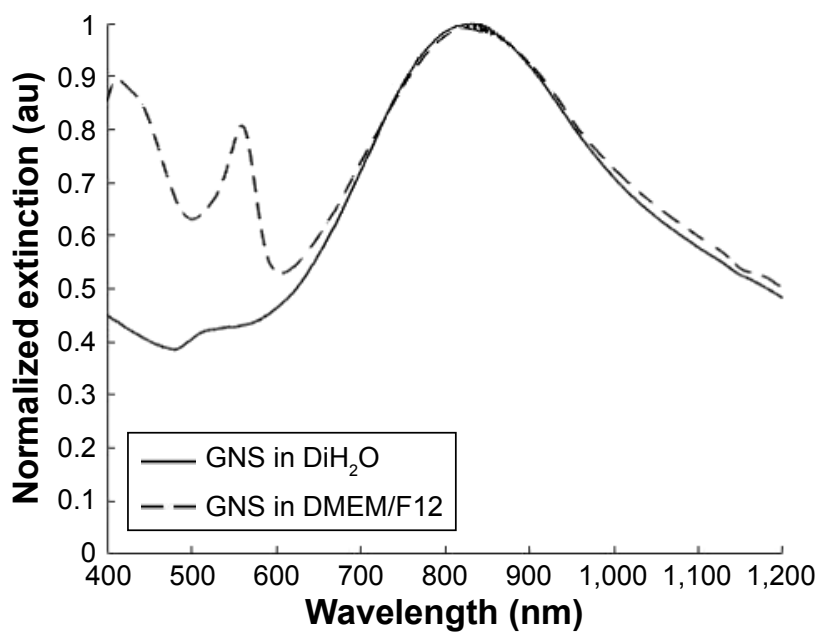

Figure SI Normalized extinction spectra of GNS in distilled water $\left(\mathrm{DiH}_{2} \mathrm{O}\right.$, solid line) and in DMEM/FI 2 media (dashed line) after 24 hours of incubation. No shift was observed for the GNS absorption peak (located at $830 \mathrm{~nm}$ ) when incubating in DMEM/FI2 for 24 hours, indicating that the nanoparticles were stable in cell culture medium. Peaks observed at $560 \mathrm{~nm}$ and $440 \mathrm{~nm}$ are due to the presence of DMEM/FI 2 medium.

Abbreviations: DMEM/FI2, Dulbecco's Modified Eagle's Medium: Nutrient Mixture F-12; GNS, gold nanostars.

\section{Publish your work in this journal}

The International Journal of Nanomedicine is an international, peerreviewed journal focusing on the application of nanotechnology in diagnostics, therapeutics, and drug delivery systems throughout the biomedical field. This journal is indexed on PubMed Central, MedLine, CAS, SciSearch $®$, Current Contents ${ }^{\circledR} /$ Clinical Medicine,
Journal Citation Reports/Science Edition, EMBase, Scopus and the Elsevier Bibliographic databases. The manuscript management system is completely online and includes a very quick and fair peer-review system, which is all easy to use. Visit http://www.dovepress.com/ testimonials.php to read real quotes from published authors. 